УДК 553.981

\title{
ОЦЕНКА ТОЧНОСТИ ОПРЕДЕЛЕНИЯ КОЭФФИЦИЕНТА ПОРИСТОСТИ ПРИ ВЫПОЛНЕНИИ ТРЕХМЕРНЫХ ГЕОЛОГИЧЕСКИХ ПОСТРОЕНИЙ
}

\author{
Пономарев Александр Иосифович 1 , \\ pnmrv@mail.ru
}

\author{
Меркулов Анатолий Васильевич2, \\ Priemnaia@ygd.gazprom.ru
}

\author{
Сопнев Тимур Владимирович ${ }^{3}$, \\ gdu@gd-urengoy.gazprom.ru
}

\section{Мурзалимов Заур Уразалиевич 2 , Z.Murzalimov@yamburg.gazprom.ru}

Кущ Иван Иванович²,

I.Kusch@yamburg.gazprom.ru

\section{Кожухарь Руслан Леонидович², R.Kozhukhar@ygd.gazprom.ru}

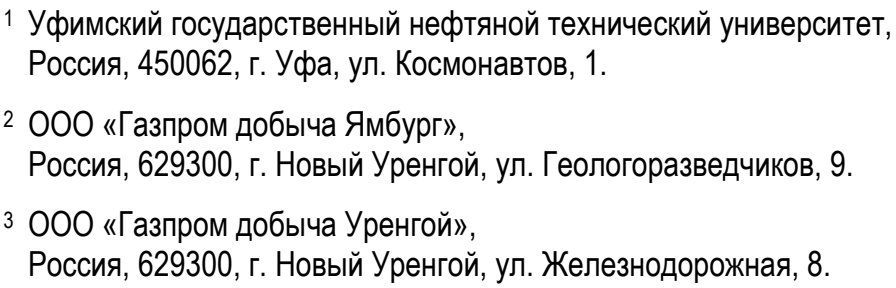

Актуальность исследования обусловлена необходимостью повышения точности прогнозирования технологических показателей разработки месторождений углеводородного сырья. Это позволит подбирать оптимальное оборудование для подготовки сырья, сдвигать сроки ввода промышленных мощностей для достижения максимального экономического эффректа от разработки месторождений.

Цель: определить точность расчета коэфффициента пористости месторождения, принятую при подсчете запасов и проектировании разработки; оценить вариации коэффиииента пористости во всем объеме месторождения; оценить степень снижения точности определения коэффрициента пористости при переходе от этапа керновых исследований до трехмерной геологической модели; установить причины снижения точности определения коэффрициента пористости при трехмерном геологическом моделировании.

Объекты: сеноманская залежь нефртегазоконденсатного месторождения на севере Западной Сибири.

Методы: вариограммный анализ, трехмерное геологическое моделирование, оценка точности определения коэффрициента nористости.

Результаты. Для корректного выполнения подсчета запасов и подготовки проектного документа требуется определить коэффрициент пористости с погрешностью не более 5 \%. В работе оценена вероятность достижения требуемого уровня точности расчета коэфффициента пористости при подсчете запасов только по данным геофизических исследований скважин, по результатам данных геофизических исследований скважин, но с учетом расположения скважин по площади месторождения, а также с учетом пространственного распространения коэфрфициента пористости. Выполнена оценка границ изменения коэфьфициента пористости по всему объему породы-коллектора, что в дальнейшем может послужить основой для адаптации гидродинамической модели. Рассчитано снижение соотношения объемов исследованного керна к объему породы по геофизическим исследованием скважины, к общему объему породы-коллектора, а также проведена оценка точности определения коэфрфициента пористости при переходе от лабораторных исследований к среднему коэфффициенту пористости по всему объему породы-коллектора.

\section{Ключевые слова:}

Запасы углеводородного сырья, подсчет запасов углеводородного сырья, геологическое моделирование, вариограммный анализ, погрешность расчета запасов углеводородов.

\section{Введение}

Определение запасов нефти и газа месторождений всегда сопряжено с долей неопределенности. Фильтрационно-емкостные свойства (ФЕС), входящие в формулу подсчета запасов объемным методом, не могут быть напрямую измерены для всего объема месторождения, поэтому значения подсчетных параметров, попадающие в заключительный отчет, рассчиты- ваются с применением множества математических операций (осреднение, аппроксимирование, взвешивание и т. д.). При выполнении подсчета запасов определяются наиболее вероятные значения подсчетных параметров и, соответственно, геологических запасов. Для геологических моделей нормативно установлено допустимое отклонение погрешности в 5 \% для всех подсчетных параметров и величины запасов. 
Допустимая погрешность в определении геологических запасов установлена одинаковой для всех месторождений и никак не изменяется в зависимости от количества и качества исходных данных. Если оценка геологических запасов выполнена неверно и превышает установленные лимиты, эта погрешность в конечном итоге начнет влиять на качество прогнозирования показателей разработки. Особенно ярко эта погрешность будет проявляться на поздних этапах разработки, поскольку остаточные запасы углеводородного сырья (УВС) снижаются, а абсолютное значение погрешности определения запасов УВС остается постоянным. Поэтому величина относительной погрешности постепенно растет, и невязка прогнозируемых показателей разработки с фактом также растет. При этом специалисты по адаптации гидродинамических моделей обязаны обеспечить допустимое отклонение по геологическим запасам УВС в гидродинамическое модели, не взирая на фактическую динамику показателей разработки месторождения.

\section{Оценка погрешности расчета коэффициента пористости}

В современной российской практике геологогидродинамического моделирования не получили широкого распространения процедуры по адаптации гидродинамических моделей с использованием геологических параметров, входящих в формулу подсчета запасов. Причина сложившейся ситуации проста - во временном регламенте [1] оценка качества построений геолого-гидродинамической модели производится в первую очередь сопоставлением созданной и адаптированной к показателям разработки математической модели к подсчету запасов. Согласно требованиям [1] по оценке качества геолого-гидродинамических моделей, допустимая погрешность в оценке запасов и всех подсчетных параметров составляет всего 5 \%. При этом не учитываются геологические факторы, которые могут повлиять на величину допустимой погрешности определения запасов. Тем самым специалисты по моделированию месторождений при адаптации фильтрационных моделей ограничены изменениями только гидродинамических характеристик (относительные фазовые проницаемости, капиллярные давления, абсолютная проницаемость и т. д.), не затрагивающих подсчетные параметры. Реальная точность определения геологических запасов может значительно отличаться от $5 \%$. В работе [2] предлагается выполнять подсчет запасов на основе нечетких множеств, и авторы приходят к выводу, что погрешность определения запасов для объемного метода составляет 20-30\%. Именно поэтому в мировой практике большую популярность приобретает интегрированное геолого-гидродинамическое моделирование [3-6]. В процессе интегрированного моделирования геологическая модель строится параллельно с гидродинамической, при этом геологическая модель строится таким образом, чтобы максимально точно воспроизвести фактические показатели разработки.

Реальная точность расчета подсчетных параметров может быть получена при анализе всех этапов подсчета запасов начиная с лабораторных исследований керна и заканчивая математическими вычислениями при построении трехмерной геологической модели. На рис. 1 представлены основные этапы возникновения погрешностей определения ФЕС в рамках подсчета запасов. Самыми достоверными данными являются лабораторные исследования керна. При этом важно отметить, что влияние гистерезиса фильтрационно-емкостных свойств кернового материала при изменении напряженного состояния выходит за рамки настоящей статьи. В данном случае на точность определения ФЕС влияет лишь погрешность лабораторных приборов и погрешность, возникающая на этапе отбора и транспортирования керна. На следующем этапе интервалам керна с одинаковыми литологическими параметрами присваивается осредненное значение подсчетного параметра нескольких образцов. Процедура осреднения подсчетных параметров, определенных по лабораторным образцам, вносит дополнительную погрешность. Кроме того, количество исследований кернового материала недостаточно для построения геологической модели, поэтому для увеличения охвата месторождения исследованиями применяют геофизические исследования скважин, которые являются косвенными методами определения емкостных свойств. Между ФЕС, определенными в лабораторных условиях, и физическими полями, наблюдаемыми геофизическими приборами, устанавливается корреляционная зависимость. Таким образом, получается большое количество исследований с повышенной погрешностью. На этом этапе возможно выполнение подсчета запасов [7-9], в ходе которого определяются эталонные значения подсчетных параметров и величины запасов УВС для месторождения. Однако только данных ГИС недостаточно для прогнозирования показателей разработки месторождения, для этого применяется геологическое и фильтрационное моделирование. При геологическом моделировании по данным ГИС определяются значения подсчетных параметров в каждой точке пространства залежи. Переход от маленького объема залежи, изученной средствами ГИС, к общему объему залежи сопровождается появлением погрешности определения подсчетных параметров в межскважинном пространстве. Суммируя все возникающие погрешности, можно рассчитать общую погрешность определения подсчетного параметра.

Величина погрешности определения подсчетных параметров керна в условиях лабораторного исследования будет зависеть от оборудования конкретной лаборатории, в целом эта погрешность незначительна по сравнению с остальными операциями, поэтому в дальнейшем не учтена. Погрешность определения подсчетного параметра при переходе от керна к ГИС оценивается коэффициентом детерминации, показывающим степень связи наблюдаемых физических полей со значениями подсчетного параметра.

Гораздо сложнее оценить погрешность при переходе от геофизических исследований к трехмерному объему залежи. Как правило, определение подсчетных параметров для всего пространства месторождения осуществляется методами геостатистики. В специализированном программном обеспечении геоло- 
гического и гидродинамического моделирования разработки месторождений УВС уделяется большое внимание задачам, связанным с выявлением и снижением неопределенностей в геологическом строении [8-13]. В геологических симуляторах большое распространение для определения геологической неопределенности получил метод «Монте-Карло» $[14,15]$. Применение данного метода подразумевает использование принятого вероятностного закона распределения геологических параметров, полученных лабораторным методом или по данным ГИС. Изменяя настройки расчета геологических параметров, определяют наиболее вероятное значение геологического параметра и его вероятностное распределение. Сложность в применении метода Монте-Карло заключается в определении диапазонов изменения параметров, участвующих при построении трехмерного распределения геологического параметра. Геологические риски, в том числе и без учета вариативности исходных данных, могут быть определены с помощью многовариантного геологического моделирования [16-18]. Подобные методики очень распространены на практике, однако для определения границ варьирования подсчетных параметров и процентилей необходимо выполнить большое количество построений геологической модели $[19,20]$. Подсчет запасов по методу материального баланса является хорошим вариантом по определению запасов для разрабатываемого месторождения $[21,22]$, однако он не позволяет оценить вариативность отдельных геологических параметров, а также данный метод неприменим до начала промышленной разработки месторождения. Альтернативным подходом к определению погрешности среднего значения геологического параметра при пространственном распределении геологического параметра является применение вариограммного анализа [23-25]. В вариограммы, используемые при построении пространственного распределения геологического параметра, заложены неопределенности, вызванные пространственной связностью геологических параметров.

Оценивая погрешность определения подсчетного параметра, рассчитывается не только вероятностная оценка величины начальных геологических запасов, но и минимальное/максимальное значение начальных геологических запасов. Расширение установленных методическими рекомендациями [1] рамок по максимально допустимому отклонению в расчете величины запасов и подсчетных параметров позволит более гибко адаптировать гидродинамическую модель к фактическим показателям разработки и, как следствие, получать более точный прогноз показателей разработки на весь период.

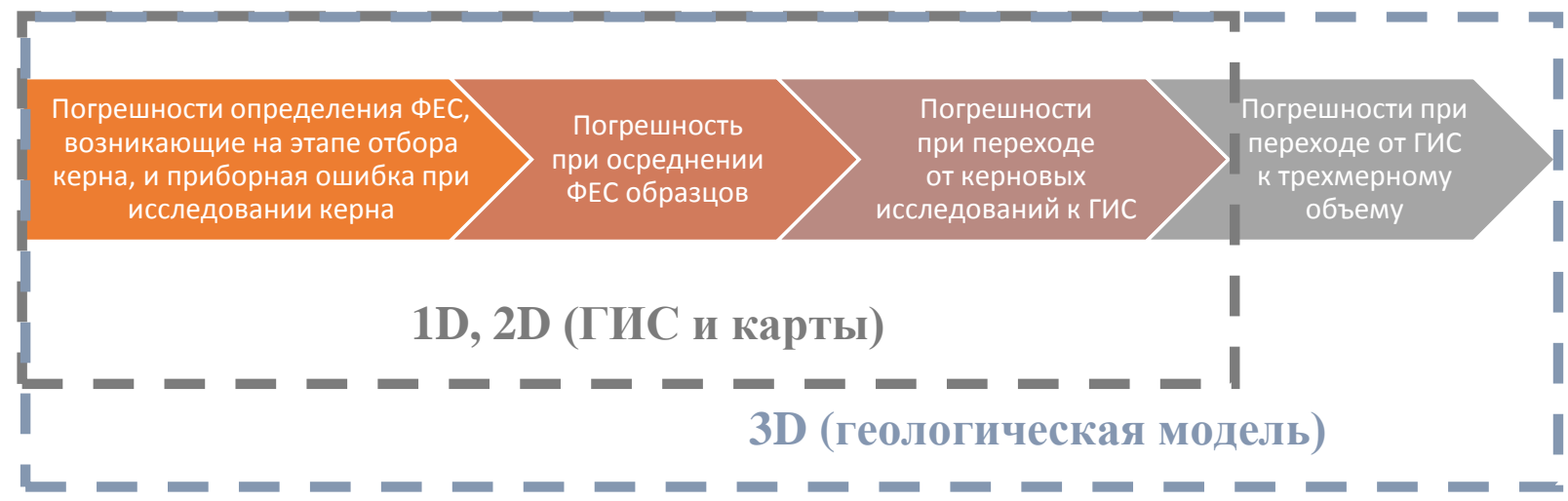

Pис. 1. Схема возникновения погрешности при определении ФЕС

Fig. 1. Scheme of error occurrence in determining the filtration-capacitive properties

Для примера проведем оценку точности определения коэффициента пористости для одного из газовых месторождений севера Западной Сибири, которое является достаточно хорошо изученным и большая часть запасов которого приходится на категорию запасов «А». Керн отобран из интервала сеноманской залежи месторождения по 21-ой скважине. Коэффициент пористости определен по зависимости от параметра $\alpha_{\text {пс }}$ (рис. 2). Нижнее граничное значение коллектора по $\alpha_{\text {пс }}$ составляет 0,21 , нижнее граничное значение пористости для коллектора составляет 0,235 д. ед. Коэффициент пористости по керну определен по 441 интервалу. Исследовано 1857 образцов из 852,3 метров эффективной толщины. Общий объем коллектора, по которому коэффициент пористости принят по результатам керновых исследований, составляет $25,86 \mathrm{~m}^{3}$.
При переходе от керна к ГИС определяется зависимость между прямым замером пористости по керну и наблюдаемым в геофизическом приборе значением физического поля. Пористость в газонасыщенной части определена по 158 скважинам, суммарная эффективная газонасыщенная толщина, по которой определен коэффициент пористости по ГИС, составляет 16745,1 м. Объем коллектора, по которому коэффициент пористости принят по ГИС, составляет $3674 \mathrm{~m}^{3}$.

Общий же объем коллектора по месторождению составляет $77661457000 \mathrm{~m}^{3}$. Таким образом, доля коллектора, по которому коэффициент пористости определен по керну, составляет 0,000000033 \%, а по ГИС-0,000004731\%. В целом такой низкий охват объема коллектора керновыми и геофизическими исследованиями характерен для всех месторождений, разрабатываемых скважинным способом. 


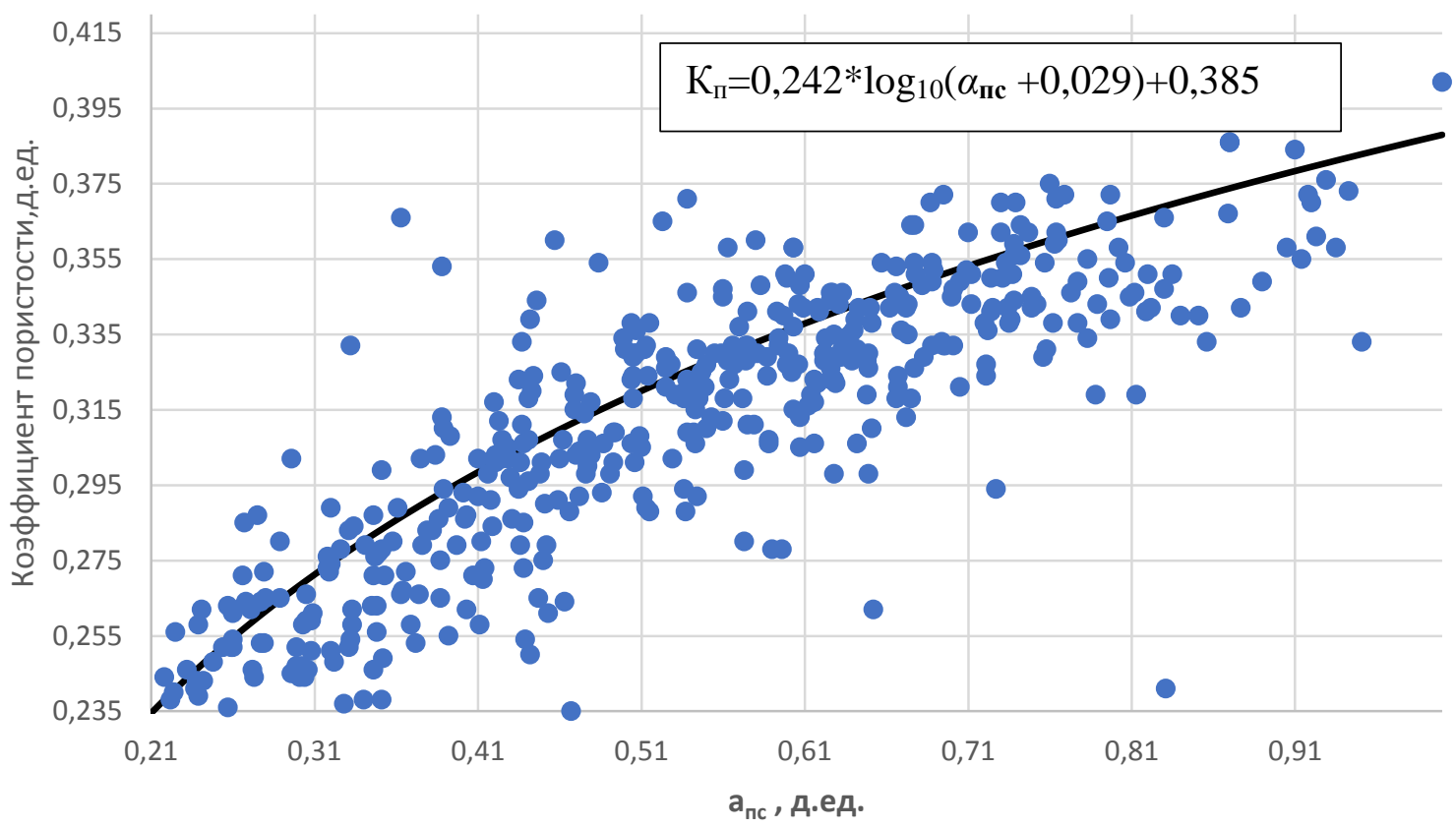

Pис. 2. Определение зависимости коэффициента пористости от $\alpha_{n c}$

Fig. 2. Determination of porosity coefficient dependence on $\alpha_{n c}$

Среднее значение пористости по керну принято равным $32,146 \%$, по ГИС в интервалах отбора керна - 33,067 \%, по ГИС по месторождению в целом $32,933 \%$. Гистограммы распределения пористости для всех случаев представлены на рис. 3 .

Несмотря на то, что точность результатов интерпретации ГИС ниже, чем по керновым исследованиям, построение геологической модели и оценка геологических запасов производится по ФЕС, определенным по ГИС. Это связано с незначительным объемом коллектора, по которому выполнены керновые исследования, к примеру, для рассматриваемого месторожде- ния объем коллектора с керновыми исследованиями в 142 раза меньше, чем объем коллектора с результатами интерпретации ГИС. Однако для дальнейших расчетов важно учитывать степень соответствия керновой пористости и результатов интерпретации ГИС, для этого определена дисперсия расхождения расчетного и кернового значения пористости. Используя нормальное распределение, определена вероятность среднего значения коэффициента пористости по данным ГИС (рис. 4). Вероятность того, что при подсчете запасов коэффициент пористости был определен с погрешностью $5 \%$, составляет $60,2 \%$.

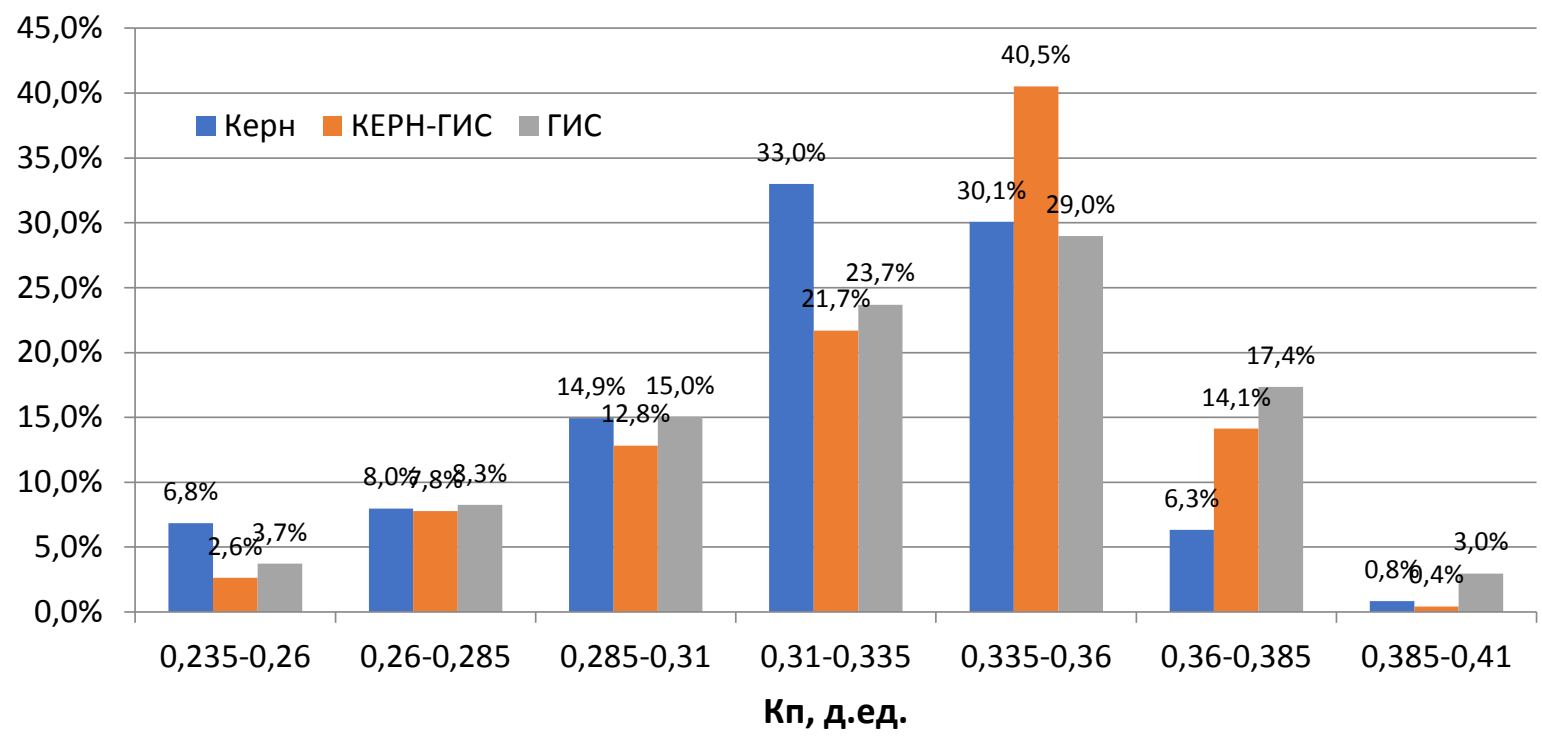

Рис. 3. Гистограммы распределения коэффициента пористости по результатам керновых исследований и геофизических исследований скважин

Fig. 3. Histograms of porosity coefficient distribution according to the results of core studies and geophysical studies of wells 


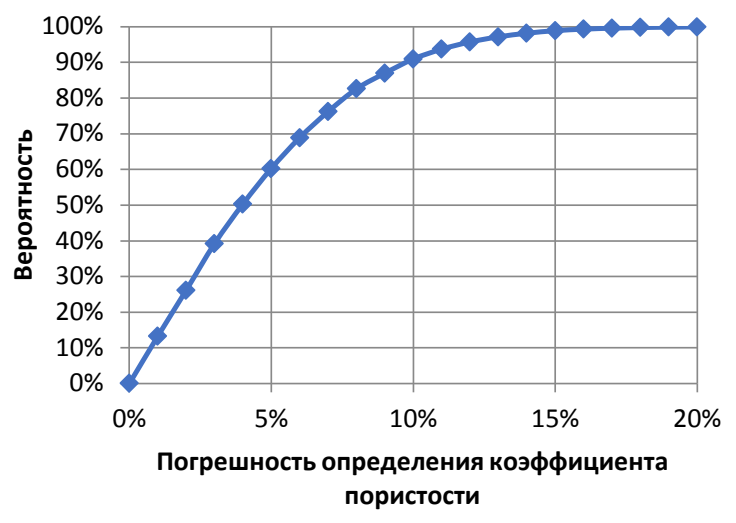

Рис. 4. График вероятности определения среднего значения коэффиииента пористости по ГИС

Fig. 4. Graph of probability of determining the average value of the porosity coefficient for geophysical studies of wells

При определении трехмерного распределения коэффициента пористости по всему объему коллектора месторождения необходимо рассчитать значение в 99,999995 \% объема залежи, по которому нет никаких исследований. Эта задача решается в процессе геологического моделирования. Наиболее распространенным методом построения трехмерных распределений геологических параметров является использование методов геостатистики, в основе которой лежит вариограммный анализ. Вариограммы позволяют оценить связанность геологических параметров в пространстве. На рис. 5 приведены вариограммы для коэффициента пористости по трем направлениям. При этом использовалась экспоненциальная вариограмма:

$$
\gamma^{\prime}(h)=\sigma^{2}\left(1-\exp \left(-\frac{h}{r / 3}\right)\right),
$$

где $r$ - ранг вариограммы; $h$ - расстояние от наблюдаемой точки до моделируемой области; $\sigma^{2}$ - вариация; $\gamma(h)$ - вариация на расстоянии $h$.

Ранги вариаограмм в параллельном, нормальном и вертикальном направлениях составляют 1050, 2000 и 22 м, соответственно. С учетом полученных вариаограмм весь объем месторождения разделяется на зоны с различной вариацией (рис. 6). На рис. 7 представлен график вероятности среднего значения коэффициента пористости с учетом вариограммного анализа. Также в вероятности на рис. 7 учтена точность расчета пористости по ГИС, определенная ранее. Для всего объема месторождения вероятность того, что коэффициент пористости определен с погрешностью $5 \%$, составляет $37,5 \%$.

В свою очередь, альтернативой вариограммному анализу может быть оценка дисперсии среднего коэффициента пористости по скважинам. Это позволит смоделировать ситуацию, в которой скважины, участвующие при подсчете запасов, располагались бы в других точках в границах площади газонасыщенности и соответственно имели бы другие средние значения коэффициента пористости. Для этого по всем сква- жинам на месторождения рассчитаны средние значения коэффициента пористости и определена дисперсия средних значений пористости по скважинам. Используя нормальное распределение, получаем вероятность среднего значения коэффициента пористости для любой гипотетической скважины в пределах контура газоносности. Дисперсия в этом случае составляет 0,000223 . При определении вероятности расчета среднего коэффициента пористости можно учесть удаленность скважин друг относительно друга - провести декластеризацию данных [26]. Самым простым решением для декластеризации будет применение диаграмм Вороного и разделение всей площади месторождения на зоны. В центре каждой зоны располагается скважина, участвующая в подсчете запасов. В каждой зоне принимается значение коэффициента пористости, равное значению по соответствующей скважине. Дисперсия среднего коэффициента пористости с учетом декластеризации составляет 0,000215 . В итоге вероятность того, что отклонение фактического коэффициента пористости от утвержденного значения будет менее $5 \%$, составляет $49,9 \%$ с учетом изменчивости коэффициента пористости по площади и $50,1 \%$ с учетом декластеризации (рис. 8 ).
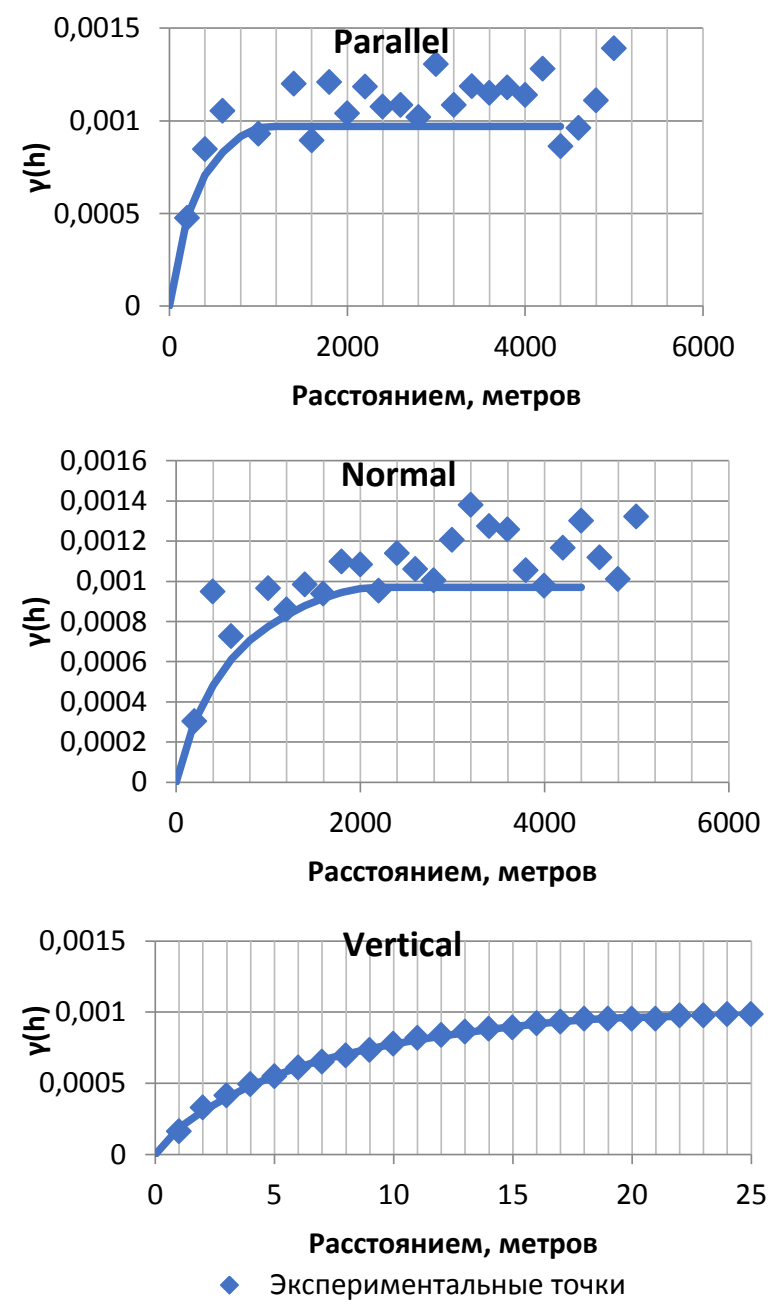

Pис. 5. Вариограммы

Fig. 5. Variograms 


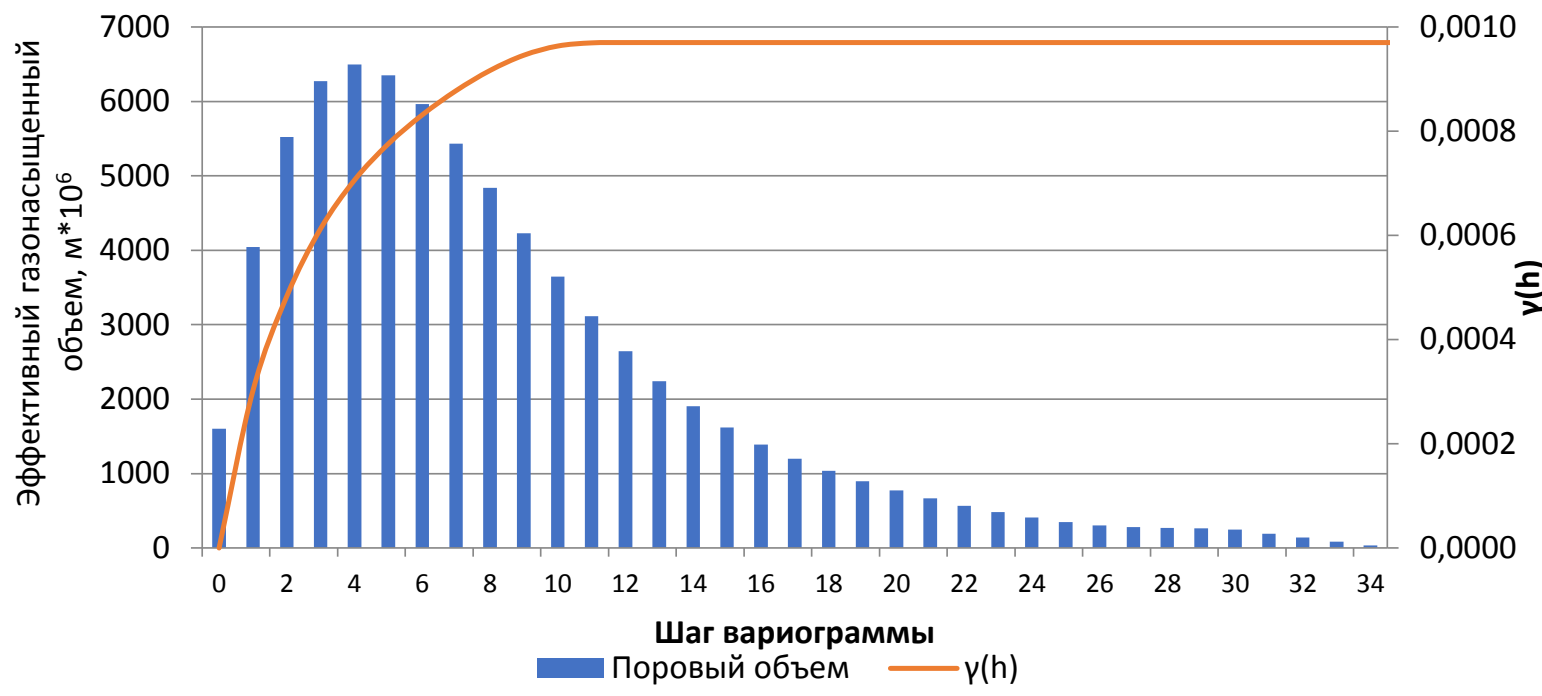

Pис. 6. Распределение эффективного порового объема с учетом шага вариограммы

Fig. 6. Distribution of the effective pore volume taking into account the step of the variograms

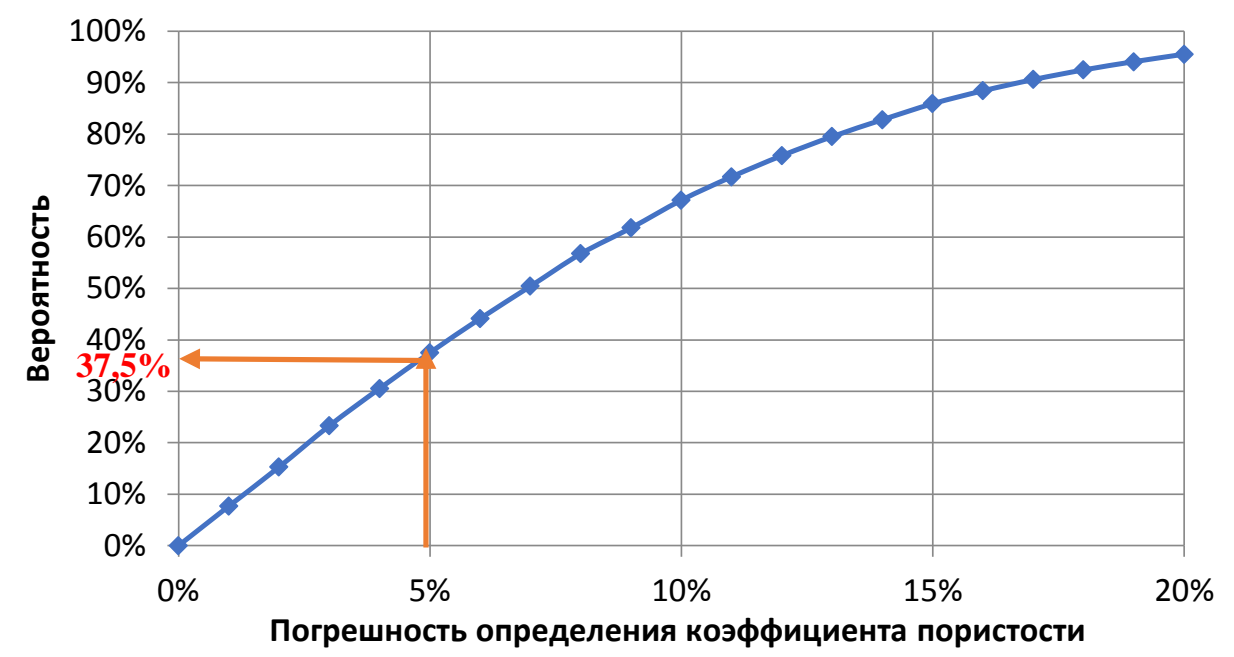

Рис. 7. График вероятности определения среднего значения коэффициента пористости по ГИС и вариограммному анализа

Fig. 7. Graph of probability of determining the average value of the porosity coefficient from geophysical studies of wells and variogram analysis

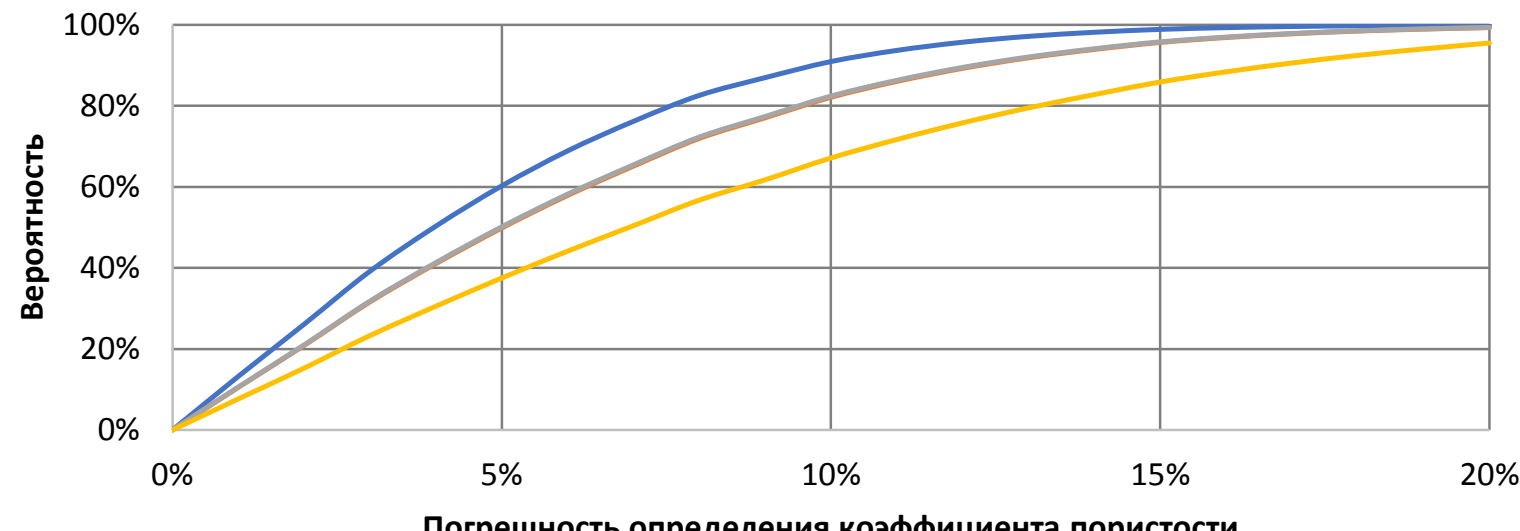

Погрешность определения коэффициента пористости

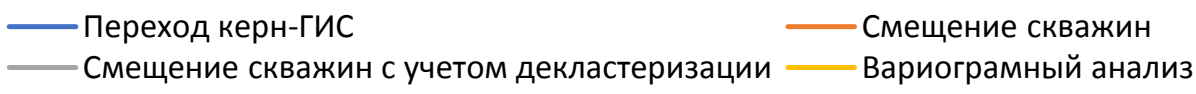

Pис. 8. График вероятности определения среднего значения коэффициента пористости

Fig. 8. Graph of probability of determining the average value of the porosity coefficient 


\section{Заключение}

Объемы горной породы, по которой проводятся определения ФЕС для выполнения подсчета запасов и проекта разработки, ничтожно малы по сравнению с общим объемом залежи. Это приводит к появлению неопределенности пространственного распределения значений подсчетных параметров, например, коэффициента пористости. При переходе от керновых измерений к геофизическим вероятность того, что погрешность определения коэффициента пористости составит менее 0,016 д. ед. (что соответствует $5 \%$ погрешности от среднего коэффициента пористости), составляет 60,2 \%. Переход к двумерной модели, с помощью определения среднего коэффициента пористости по

\section{СПИСОК ЛИТЕРАТУРЫ}

1. Временный регламент оценки качества и приемки трехмерных цифровых геолого-гидродинамических моделей, представляемых пользователями недр в составе технических проектов разработки месторождений углеводородного сырья на рассмотрение ЦКР Роснедр по УВС, от 19.04.2012.

2. Алтунин А.Е., Семухин М.В. Расчеты в условиях риска и неопределенности в нефтегазовых технологиях: монография. Тюмень: Изд-во Тюменского государственного университета, 2004. - $296 \mathrm{c}$.

3. Integration of petroleum and reservoir engineering workflows in production database management systems / D. Talbot, P. Jeffs, A. Hyde, A. Kohok, D. Rossi // Society of Petroleum Engineers. 2016. - SPE-181071-MS. - 13 p.

4. Burgstaller C. New approaches of using fluid level data for production optimization and reservoir engineering applications // Society of Petroleum Engineers. - 2016. - SPE-180159-MS. - 10 p.

5. Appraising carbon geological-storage potential in unconventiona reservoirs: engineering-parameters analysis / Zhiming Chen, Xinwei Liao, Xiaoliang Zhao, Xiaojiang Li // Society of Petroleum Engineers. - 2018. - SPE-189442-PA. - 13 p.

6. An integrated geological-reservoir engineering technique for in creasing confidence in field development planning for a reservoi under primary depletion / Aakash Biswas, Ali Al Jumah, Farhad Lalji, Mohamad Doush // Society of Petroleum Engineers. - 2017. SPE-188780-MS. - 13 p.

7. Петерсилье В.И., Пороскуна В.И., Яценко Г.Г. Методические рекомендация по подсчету геологических запасов нефти и газа объемным методом - М.; Тверь: ВНИГНИ, НПЦ «Тверьгеофизика», 2003. - 259 с.

8. Сарваретдинов Р.Г., Гильманова Р.Х., Миннуллин А.Г. К вопросу о потере запасов нефти при традиционном способе подсчета // Нефтепромысловое дело. - 2019. - № 2. - С. 33-36

9. Кирсанов А.Н., Зинченко И.А., Кирсанов С.А. Технология подсчета и дифференциации запасов газа по качеству терригенных коллекторов // Газовая промышленность. - 2004. № $10 .-$ C. $28-34$.

10. Файзуллина С.Р., Сафаров А.Ф., Михайлов С.Н. Особенности современного подхода подсчета запасов с применением трехмерного геологического моделирования на примере массивных залежей нефтяного месторождения // Нефтяная провинция. - 2017. - № 4 (12). - С. 83-90.

11. Громов Е.В., Торопов Д.А. Повышение точности подсчета запасов с применением 3D моделирования (на примере месторождения «Партомчорр») // Горный информационноаналитический бюллетень (научно-технический журнал). 2017. - № 23. - С. 158-166.

12. Ядрышникова О.А., Алтунин А.Е Комплекс программ для оценки запасов углеводородов и подсчетных параметров в условиях неопределенности // Вестник тюменского государственного университета. Физико-математическое моделирование. Нефть, газ, энергетика. - 2018. - Т. 4. - № 4. C. $249-265$. скважинам с учетом и без учета декластеризации, составляет уже 49,9 и 50,1 \% соответственно. А при переходе к трехмерной геологической модели вероятность того, что для всего объема залежи погрешность определения не превышает 5 \%, составляет $37,5 \%$.

Можно применить различные методики оценки точности определения подсчетных параметров, однако выводы от этого не изменятся: повышение точности определения подсчетных параметров возможно только в случае повышения количества исследований при увеличении плотности сетки разбуривания месторождения, либо за счет промысловой информации о динамике разработки месторождения при построении/актуализации геологической модели.

13. Kupfersbcrgcr H., Dcutsch C.V. Methodology for integrating analogue geologic data in 3-d variogram modeling // AAPG Bidliten. 1999. - № 83 (8). - Р. 1262-1278.

14. Смолич С.В., Смолич К.С. Решение горно-геологических задач методом «Монте-Карло». - Чита: ЧитГУ, 2004. - 103 с.

15. Kelliher C.F., Mahoney L.S. Using Monte Carlo simulation to improve long-term investment decision // The Appraisal Journal. 2000. - № 1. - P. 44-56.

16. Dcutsch C.V., Joumcl A.G. Stochastic modeling and geostatistics: principles, methods, and case studies // AAPG Computer Applications in Geology. - 1995. - № 3. - P. 131-142.

17. Dculsch C.V., Srinivasan S. Improved reservoir management through ranking stochastic reservoir models // SPE/DOE Tenth Symposium on Improved Oil Recovery. - Tulsa, OK, 1996. - SPE Paper Number 35 411. - P. 105-113.

18. Dcutsch C.V., Wang L. Hierarchical object-based stochastic modeling of fluvial reservoirs // Math Geology. - 1996. - № 28 (7). P. $857-880$.

19. Нугманов Б.Х., Эминов А.Ш., Рагимов Ф.В.3. Анализ чувствительности и оценка геологических рисков при подсчете запасов месторождения «Каламкас» // Научные труды НИПИ нефтегаз гнкар. - 2017. - № 3. - С. 4-8.

20. Алтунин А.Е, Семухин М.В., Ядрышникова О.А. Вероятностные и нечеткие модели оценки неопределенностей и рисков при подсчете запасов углеводородов // Вестник тюменского государственного университета. Физико-математическое моделирование. Нефть, газ, энергетика. - 2017. - Т. 3. - № 2. C. $85-99$.

21. Воронков А.А., Журова Е.Л. Системный подход к применению уравнения материального баланса для газовых месторождений // Газовая промышленность. - 2012. - № 11 (682). C. $37-41$.

22. Оценка достоверности результатов подсчета запасов газа разбуренного участка агкм / Г.П. Косачук, В.Ф. Перепеличенко, Ф.Р. Билалов, Н.П. Мартынова // Газовая промышленность. 2004. - № 1. - С. 56-58.

23. Дойч К.В. Геостатистическое моделирование коллекторов. М.; Ижевск: Институт компьютерных исследований, 2011. $400 \mathrm{c}$.

24. Gringarton E., Dcutsch C. Methodology for variogram interpretation and modeling for improved petroleum reservoir characterization // SPE Annual Technical Conference and Exhibition Formation Evaluation and Reservoir Geology. - 1999. - SPE paper Number 56654. - P. 151-163.

25. Joumel A.G., Huijbregts C.J. Mining geostatistics. - New York: Academic Press, 1978. - 600 p.

26. Вестник Roxar 2015 // Roxar. 2015. URL: http://roxar.ru/wpcontent/uploads/2017/04/\%D0\%92\%D0\%B5\%D1\%81\%D1\%82\% D0\%BD\%D0\%B8\%D0\%BA-Roxar$\% \mathrm{D} 0 \% 9 \mathrm{D} \% \mathrm{D} 0 \% \mathrm{BE} \% \mathrm{D} 1 \% 8 \mathrm{~F} \% \mathrm{D} 0 \% \mathrm{~B} 1 \% \mathrm{D} 1 \% 80 \% \mathrm{D} 1 \% 8 \mathrm{C}-$ 2015.pdf (дата обращения 16.06.2020)

Поступила 17.06.2020 г. 


\section{Информация об авторах}

Пономарев А.И., доктор технических наук, профессор, заведующий кафедрой разработки и эксплуатации газовых и газоконденсатных месторождений Уфимского государственного нефтяного технического университета.

Меркулов А.В., главный геолог, заместитель генерального директора ООО «Газпром добыча Ямбург».

Cопнев T.B., главный геолог, заместитель генерального директора ООО «Газпром добыча Уренгой».

Мурзалимов 3.У., начальник управления геологии, разработки и лицензирования месторождений ООО «Газпром добыча Ямбург».

Кущ И.И., начальник геологического отдела ООО «Газпром добыча Ямбург».

Кожухарь Р.Л., ведущий геолог ООО «Газпром добыча Ямбург». 
UDC 553.981

\title{
ACCURACY OF POROSITY, WHEN PERFORMING THREE-DIMENSIONAL GEOLOGICAL IMAGES
}

\author{
Alexander I. Ponomarev 1 , \\ pnmrv@mail.ru
}

\author{
Anatoly V. Merkulov², \\ Priemnaia@ygd.gazprom.ru \\ Timur V. Sopnev ${ }^{3}$, \\ gdu@gd-urengoy.gazprom.ru
}

Zaur U. Murzalimov², Z.Murzalimov@yamburg.gazprom.ru

Ivan I. Kushch², I.Kusch@yamburg.gazprom.ru

\author{
Ruslan L. Kozhukhar2, \\ R.Kozhukhar@ygd.gazprom.ru \\ 1 Ufa State Oil Technical University, \\ 1, Kosmonavtov street, Ufa, 450062, Russia. \\ 2 LLC «Gazprom dobycha Yamburg», \\ 9, Geologorazvedchikov street, Novy Urengoy, 629300, Russia. \\ 3 LLC «Gazprom dobycha Urengoy», \\ 8, Zheleznodorozhnaya street, Novy Urengoy, 629300, Russia.
}

The relevance of the study is caused by the need to improve the accuracy of forecasting technological indicators of development of hydrocarbon deposits. This will allow us to select the optimal equipment for preparation of raw materials, to shift the timing of commissioning of industrial capacities, to achieve the maximum economic effect from the development of deposits.

The aim of the research is to determine the accuracy of calculation of the porosity coefficient of the field, adopted in the calculation of reserves and design development; to estimate the variation of the porosity coefficient in the entire volume of the deposit; to assess the degree of decrease in the accuracy of determining the porosity coefficient during the transition from the stage of core studies to a threedimensional geological model; to establish the reasons of decrease in accuracy of determination of porosity coefficient at threedimensional geological modeling.

Object: Cenomanian reservoir oil and gas condensate field located in the North of Western Siberia.

Methods: variogram analysis, three-dimensional geological modeling, estimation of porosity coefficient determination accuracy.

Results. For correct calculation of stocks and preparation of the project document it is required to define porosity coefficient with an error no more than $5 \%$. The authors have estimated the probability of achieving the required level of accuracy in calculation of porosity when assessing the reserves only according to geophysical researches of wells, the results of geophysical researches of wells data, but given the location of wells in the field area and also taking into account the spatial distribution of porosity. The boundaries of the porosity coefficient change in the entire volume of the deposit were estimated. This can serve as a basis for adaptation of the hydrodynamic model in the future. The ratio of the volume of the studied core to the volume of the studied geophysical researches of wells rock to the total volume of the reservoir rock is calculated, and the accuracy of determining the porosity coefficient during the transition from laboratory studies to the average porosity coefficient in the field volume is estimated.

\section{Key words:}

Hydrocarbon reserves, hydrocarbon reserves calculation, geological modeling, variogram analysis, hydrocarbon reserves calculation error.

\section{REFERENCES}

1. Vremenny reglament otsenki kachestva i priemki trekhmernykh tsifrovykh geologo-gidrodinamicheskikh modeley, predstavlyaemykh polzovatelyami nedr $v$ sostave tekhnicheskikh proektov razrabotki mestorozhdeniy uglevodorodnogo syrya na rassmotrenie CKR Rosnedr po UVS [Temporary regulations for quality assessment and acceptance of three-dimensional digital geological and hydrodynamic models submitted by subsoil users as part of technical projects for the development of hydrocarbon deposits for consideration by the Central Committee of Rosnedra on HCR] Dated 19.04.2012.

2. Altunin A.E., Semukhin M.V. Raschet $v$ usloviyakh riska i neopredelennosti $v$ neftegazovykh tekhnologiyakh: monografiya. [Calculations under risk and uncertainty in oil and gas technologies:
Monograph.] Tyumen, Tyumen State University Press, 2004. 296

3. Talbot D., Jeffs P., Hyde A., Kohok A., Rossi D. Integration of petroleum and reservoir engineering workflows in production database management systems. Society of Petroleum Engineers, 2016, SPE-181071-MS, $13 \mathrm{p}$.

4. Burgstaller C. New approaches of using fluid level data for production optimization and reservoir engineering applications. Society of Petroleum Engineers, 2016, SPE-180159-MS, $10 \mathrm{p}$.

5. Zhiming Chen, Xinwei Liao, Xiaoliang Zhao, Xiaojiang Li. Evaluating carbon geological-storage potential in unconventional reservoirs: engineering-parameters analysis. Society of Petroleum Engineers, 2018, SPE-189442-PA, 13 p. 
6. Aakash Biswas, Ali Al Jumah, Farhad Lalji, Mohamad Doush. An integrated geological-reservoir engineering technique for increasing confidence in field development planning for a reservoir under primary depletion. Society of Petroleum Engineers, 2017, SPE188780-MS, $13 \mathrm{p}$.

7. Petersile V. I., Poroskun V. I., Yatsenko G. G. Metodicheskie rekomendatsii po podschetu geologicheskikh zapasov nefti $i$ gaza obemnym metodom [Methodical recommendations for calculation of geological reserves of oil and gas volumetric method]. Moscow, Tver, VNIGNI, NPTS «Tvergeofizika», 2003. 259 p.

8. Sarvaretdinov R.G., Gilmanova R.H., Minnullin A.G. On the issue of loss of oil reserves in the traditional method of counting. Oilfield business, 2019, no. 2, pp. 33-36. In Rus.

9. Kirsanov A.N., Zinchenko I.A., Kirsanov S.A. Technology of calculation and differentiation of gas reserves by quality of terrigenous reservoirs. Gas industry, 2004, no. 10, pp. 28-34. In Rus.

10. Faizullina S.R., Safarov A.F., Mikhailov S.N. Features of modern approach of calculation of reserves with application of threedimensional geological modeling on an example of massive deposits of an oil field. Oil province, 2017, no. 4 (12), pp. 83-90. In Rus.

11. Gromov E.V., Toropov D.A. Improving the accuracy of reserves calculation using 3D modeling (on the example of the Partomchorr field). Mining information and analytical Bulletin (scientific and technical journal), 2017, no. 23, pp. 158-166. In Rus.

12. Yadryshnikova O.A., Altunin A.E. Complex of programs for estimation of hydrocarbon reserves and calculation parameters under uncertainty. Bulletin of Tyumen state University. Physical and mathematical modeling. Oil, gas, and energy, 2018, vol. 4, no. 4, pp. 249-265. In Rus.

13. Kupfersbcrgcr H., Dcutsch C.V. Methodology for integrating analog geological data in 3-d variogram modeling. AAPG Bidliten, 1999, no. 83 (8), pp. 1262-1278.

14. Smolich S.V., Smolich K.S. Reshenie gorno-geologicheskikh zadach metodom «Monte-Karlo» [Solution of mining and geological problems by «Monte Carlo» method]. Chita, Chitgu Publ., 2004. 103 p.

15. Kelliher C.F., Mahoney L.S. Using Monte Carlo simulation to improve long-term investment decision. The Appraisal Journal, 2000 no. 1 , pp. 44-56.

16. Dcutsch C.V., Joumcl A.G. Stochastic modeling and geostatistics: principles, methods, and case studies. AAPG Computer Applications in Geology, 1995, no. 3, pp. 131-142.
17. Dulsch C.V., Srinivasan S. Improved reservoir management through ranking stochastic reservoir models. SPE/DOE Tenth Symposium on Improved Oil Recovery. Tulsa, OK, 1996. SPE Paper Number 35 411, pp. 105-113.

18. Dcutsch C.V., Wang L. Hierarchical object-based stochastic modeling of fluvial reservoirs. Math Geology, 1996, no. 28 (7), pp. 857-880.

19. Nugmanov B.Kh., Eminov A.Sh., Ragimov F.V. Sensitivity analysis and assessment of geological risks in the calculation of reserves of the Deposit «Kalamkas». Scientific works of NIPI Neftegaz SOCAR, 2017, no. 3, pp. 4-8. In Rus.

20. Altunin A.E., Semukhin M.V., Yadryshnikova O.A. Probabilistic and fuzzy models of estimation of uncertainties and risks at calculation of hydrocarbon reserves. Bulletin of Tyumen state University. Physical and mathematical modeling. Oil, gas, and energy, 2017, vol. 3, no. 2, pp. 85-99. In Rus.

21. Voronkov A.A., Zhurova E.L. System approach to application of the equation of material balance for gas fields. Gas industry, 2012, vol. 682, no. 11, pp. 37-41. In Rus.

22. Kosachuk G.P., Perepelichenko V.F., Bilalov F.R., Martynova N.P. Assessment of reliability of results of calculation of reserves of gas of the drilled site of agkm. Gas industry, 2004, no. 1, pp. 56-58. In Rus.

23. Deutsch K.V. Geostatisticheskoe modelirovanie kollektorov [Geostatistical modeling of reservoirs]. Moscow, Izhevsk, Institute of computer research Publ., 2011. 400 p.

24. Gringarton E., Deutsch C. Methodology for variogram interpretation and modeling for improved reservoir characterization petroleum. SPE Annual Technical Conference and Exhibition Formation Evaluation and Reservoir Geology, 1999, SPE paper Number 56654, pp. 151-163

25. Joumel G., Huijbregts C.J. Mining geostatistics. New York, Academic Press, 1978. $600 \mathrm{p}$

26. Bulletin of Roxar. 2015. Available at: http://roxar.ru/wpcontent/uploads/2017/04/\%D0\%92\%D0\%B5\%D1\%81\%D1\%82\% D0\%BD\%D0\%B8\%D0\%BA-Roxar-\%D0\%9D\%D0\%BE\%D1\% 8F\%D0\%B1\%D1\%80\%D1\%8C-2015.pdf (accessed 16 June 2020).

Received: 17 June 2020.

\section{Information about the authors}

Alexander I. Ponomarev, Dr. Sc., professor, head of the Department, Ufa State Oil Technical University.

Anatoly V. Merkulov, chief geologist, deputy general director, LLC «Gazprom dobycha Yamburg».

Timur V. Sopnev, chief geologist, deputy general director, LLC «Gazprom dobycha Urengoy».

Zaur U. Murzalimov, head of the Department, LLC «Gazprom dobycha Yamburg».

Ivan I. Kusch, head of geological Department, LLC «Gazprom dobycha Yamburg».

Ruslan L. Kozhukhar, leading geologist, LLC «Gazprom dobycha Yamburg». 